\title{
Efectos psicológicos de femicidio en pobladores del barrio Cristo Rey, municipio de Tipitapa-Managua (estudio de casos)
}

Criselda Calero, Sayda González y Anameyling Guevara

\begin{abstract}
Resumen
El presente estudio tiene por objetivo, analizar desde el punto de vista psicológico los efectos que tiene en la población los casos de femicidio. En el barrio Cristo Rey han sucedido alrededor de tres casos de femicidio pero solo uno ha sido tipificado como tal. Este contexto hizo que se escogiera este barrio como universo de estudio con el fin de generar una reflexión introspectiva y una discusión analítica acerca de la violencia, falta de equidad de género y el fenómeno de femicidio.

El fenómeno de femicidio describe las conductas psicológicas respecto al tema de género, partiendo de patrones de comportamientos construidos desde la familia. Estos tienen como características principales: estilos de crianza, cultura patriarcal, religión y otras variables estudiadas en esta investigación.

Entre los resultados más importantes obtenidos en este estudio, está la información obtenida de los pobladores, acerca de las variables psicosociales que intervienen en la violencia contra la mujer. Entre las, más destacadas se encuentran: machismo, cultura patriarcal y celos. Todos ellos considerados como factores desencadenantes del femicidio.
\end{abstract}

Palabras clave: Femicidio, Violencia de género, Machismo, Misoginia.

\section{Introducción}

El femicidio en Nicaragua es una problemática social que va en aumento día a día; sobre ello, podemos escuchar u observar a través de los distintos medios de comunicación, cómo el hombre priva de vida a la mujer por distintas razones, ya sea por celos, infidelidad, ideas misóginias, entre muchas otras. Tal situación no solo genera pérdidas de miles de vidas de madres, esposas, hijas, sino también causa un profundo dolor en las familias, amigos y personas que habitan en la comunidad donde vivió la víctima u ocurrió el caso de femicidio. Aunque muchos de los casos quedan en impunidad, las consecuencias del mismo están presentes y son devastadoras en la familia, comunidad y sociedad.

La presente investigación se refiere a los efectos psicológicos de casos de femicidio en pobladores del barrio Cristo Rey, municipio de Tipitapa, departamento de Managua. En este trabajo se describen las conductas psicológicas respecto al tema de género en los patrones de comportamientos y cultura patriarcal construidos en las familias del barrio en estudio.

La importancia de este trabajo radica principalmente en conocer el impacto a nivel psicológico de los casos de femicidios, también presenta un breve detalle de los resultados obtenidos del trabajo de campo, las encuestas y las observaciones realizadas en el barrio Cristo Rey.

\section{Diseño metodológico}

El estudio presenta un enfoque cuantitativo. Está basado principalmente en un instrumento de medición para la recolección y análisis de los resultados. El tipo de estudio es descriptivo porque se obtuvo información 
del comportamiento de los pobladores ante el caso de femicidio en el contexto de estudio. También, es importante resaltar que este es un estudio transversal porque los resultados se manifestaron en una misma línea de tiempo, lo cual permitió ubicar de forma secuencial los principales hallazgos sobre el desarrollo del tema en estudio durante el periodo mayo- septiembre 2015.

\section{Población y muestra}

El barrio Cristo Rey cuenta con una población de 13,250 habitantes. Para esta investigación se tomó una muestra por conveniencia, la cual es una técnica no probabilística en la que los sujetos fueron elegidos programando algunos criterios de selección: aquellos pobladores que fuesen residentes del barrio, presentaran una edad del rango 1865 años y que tuvieran la disponibilidad de colaborarnos.

\section{Método cuantitativo}

En el enfoque cuantitativo se utilizó el método de la encuesta, los ítems de las preguntas tuvieron un valor definido por la escala Likert, con tópicos sobre la violencia a la mujer, machismo, cultura patriarcal, desintegración familiar y dependencia emocional. A partir de los datos recolectados, se diseñó la base de datos correspondientes, utilizando el software estadístico SPSS (Paquete Estadístico Para las Ciencias Sociales). Una vez que se realizó el control de calidad de los datos registrados, se procedió al análisis estadístico pertinente.

\section{Discusión}

La necesidad de investigar más sobre el femicidio surge de la frecuencia de la muerte de las mujeres, situación que afecta a la sociedad, comunidad, barrio, familia en donde se ha manifestado tal acto de violencia. Por lo tanto, el interés de estudiar dicha problemática es conocer a profundidad y brindar información de cómo el caso de femicidio ocurrido en el barrio Cristo Rey pudo incidir en la conducta de los pobladores.

\section{Análisis de resultados}

El enfoque cuantitativo de la investigación permitió la comprobación de las preguntas directrices, a partir de cada una de las encuestas realizadas a los pobladores del barrio Cristo Rey del municipio de Tipitapa. A su vez, se construyeron capítulos de las diferentes dimensiones para una mejor explicación de lo abordado en la presente investigación. Los hallazgos obtenidos proveen estimaciones a nivel de barrio acerca de los efectos psicológicos en los pobladores provocados por el caso de femicidio.

Los principales resultados de la investigación son los siguientes:

En el primer capítulo se contemplan los aspectos socios demográficos en los cuales destacan: la escolaridad: La escolaridad de un individuo es fundamental para el desarrollo de una sociedad, puesto que es un proceso de socialización mediante el cual la persona adquiere conocimientos que influyen en su forma de pensar y actuar. Es a través de la educación que se transmiten habilidades, valores, aprendizajes, creencias y hábitos. Por ende, la educación puede provocar un cambio de comportamiento aprendido de generaciones anteriores en el individuo.

Los pobladores del barrio Cristo Rey presentan un nivel educativo bajo: $40 \%$ educación primaria para las mujeres y 53\% educación secundaria para los hombres. Esto por consiguiente, los incapacita para obtener mejores oportunidades de trabajo y por ende mejores condiciones de vida. También, una escolaridad baja limita a los pobladores a tener diferentes inclinaciones e ideología de vida referente al futuro. Cuanta más educación tenga una persona, mejor se desenvolverá en su entorno y en muchos aspectos de su vida. 
Gráfica 1. Escolaridad.

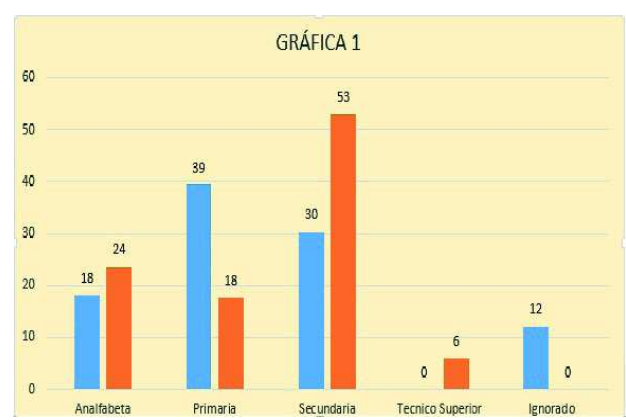

Seguidamente, en el segundo capítulo sobre la apreciación de la población sobre las funciones y tareas del hogar respecto al género sobresalen las tareas domésticas, de las cuales algunas personas consideran que son exclusivamente para las mujeres. En el barrio Cristo Rey el $70 \%$ de las mujeres y el $53 \%$ de los hombres piensan que "las tareas domésticas no son exclusivamente para las mujeres". Lamentablemente, el resto de los encuestados tanto hombres como mujeres, siguen teniendo una idea errada en la que el hombre y la mujer tienen roles muy específicos, mismas que solo causan diferencias entre el género.

En cada una de las familias, comunidades y departamentos, la educación, el tipo de cultura a la cual pertenecen o el contexto histórico en que se desarrollan han creado diferentes normas, tareas, acciones, actividades que están dirigidas a un género específico, prefigurando así una posición en la estructura social que se atribuyen y son asumidas diferencialmente por mujeres y hombres.

Grafica 2. Las tareas domésticas son exclusivamente para las mujeres.

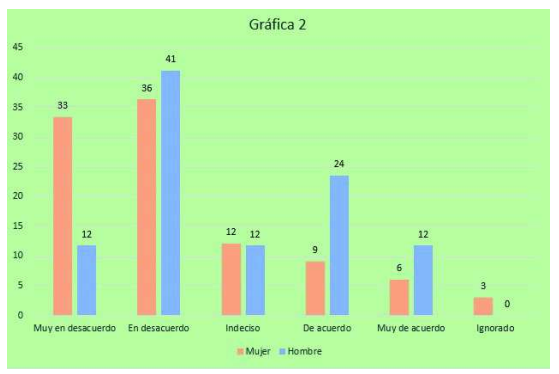

Criselda Calero, Sayda González y Anameyling Guevara
Posteriormente, en el capítulo tres acerca del maltrato y femicidio, según la población en estudio se destacan las justificaciones sobre la violencia de género. En la encuesta se les preguntó a los pobladores del barrio Cristo Rey cuáles eran las razones por las que el hombre puede asesinar a una mujer, observándose la postura de los hombres con un $47 \%$, de que la razón principal fueron los "celos"; en tanto, esta misma opción la asumió el $45 \%$ de las mujeres.

Estos resultados demuestran que tanto hombres como mujeres justifican la violencia y no porque lo crean así, sino por la educación $\mathrm{y}$ las influencias sociales que pudo tener y en las que se encuentran. Si se continúa pensando de esta manera, los que cometen este acto siempre tendrán una excusa y nunca enfrentarán su responsabilidad.

Grafica 3. Razones mencionadas por las y los entrevistados por las que el hombre puede asesinar a una mujer.

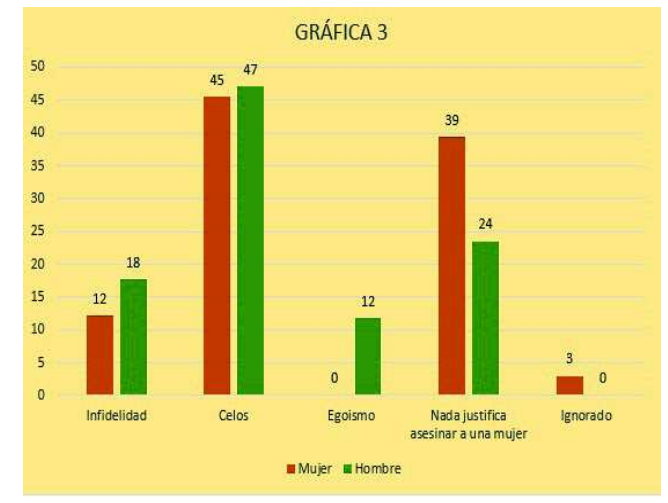

Otro punto importante a destacar son las causas vinculadas a la tolerancia y legitimación de la violencia. En relación a los motivos por los que la mujer soporta violencia dentro del hogar, en el caso de las mujeres, el $27 \%$ respondió como motivo para soportar violencia, "mantener unido el hogar" y más de la mitad de los hombres (53\%) dijo que por miedo.

Los resultados de esta interrogante evidencian que las mujeres aceptan que 
pueden llegar a soportar violencia por razones inculcadas dentro del ambiente familiar en el que crecieron. También se relacionan con otros aspectos como miedo a la pareja, dependencia económica y por no querer causar ningún dolor a sus hijos.

Grafica 4. Motivos por los que la mujer soporta violencia dentro del hogar.

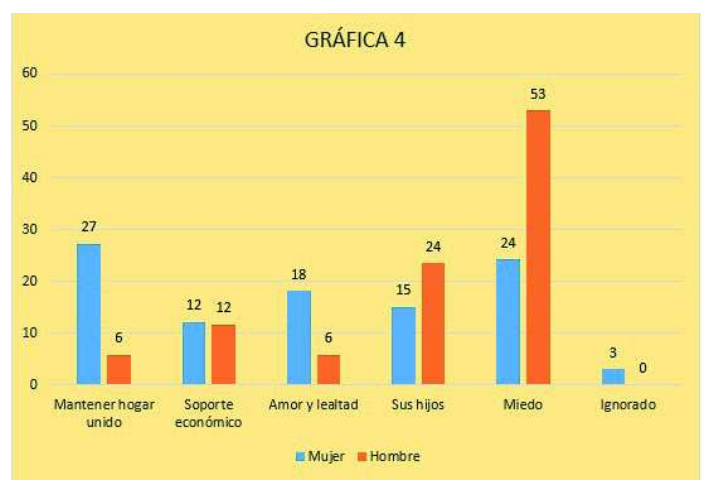

Finalmente, elcuartocapítulo sobrelos efectos psicológicos que provoca el femicidio en los pobladores del barrio Cristo Rey responde a que el femicidio no concluye solo con la muerte violenta de la mujer, sino que genera un gran impacto que puede influir en lo emocional, comportamental y pensamientos de las familias, amigos y comunidad, provocando efectos perjudiciales en ellos.

En este capítulo se analizó quiénes son los más vulnerables ante un caso de femicidio. De esto se obtuvo que el 55\% de las mujeres considera que los más vulnerables son los hijos, quienes tras la muerte de una mujer en la mayoría de los casos, sus madres, quedan expuestos a un destino incierto que puede repercutir en muchos ámbitos de sus vidas, tomando un patrón de comportamiento negativo.

Por su parte, los hombres, representados con el $47 \%$, opinan que la mayor afectación se daría en la comunidad, porque puede implicar un aprendizaje de conducta desfavorable para los niños, quienes en un futuro podrían repetir el mismo círculo de violencia hacia las mujeres, creando un entorno de inseguridad para todos los habitantes.

Gráfica 5. Afectados respecto a casos de femicidio.

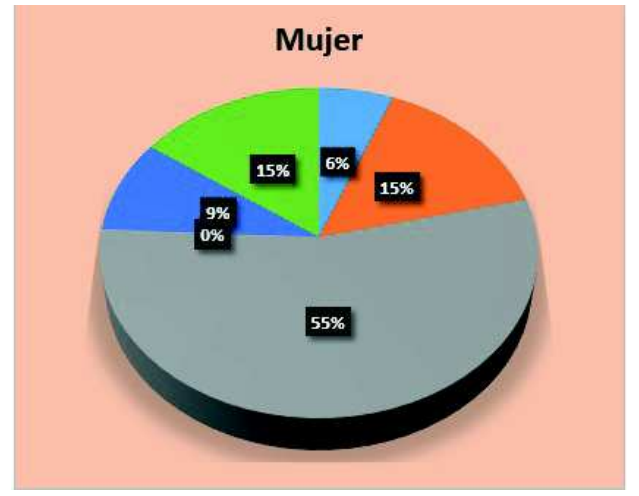

Gráfica 6.

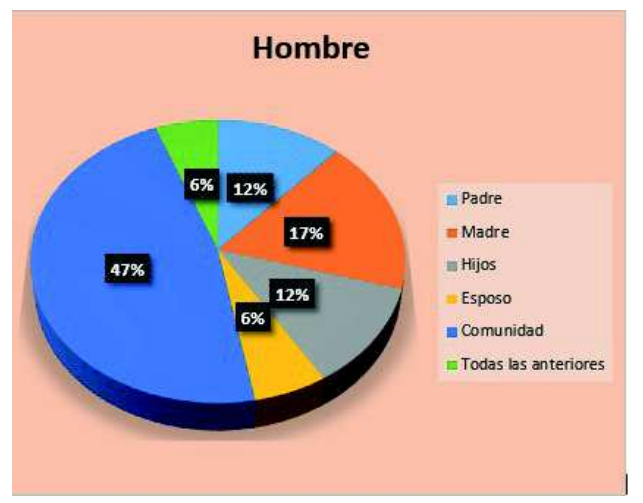

Otro inciso a destacar en este cuarto capítulo son las afectaciones por femicidio en el barrio Cristo Rey. En este sentido, se comprendió que el femicidio es un fenómeno de orden social, en el cual sus efectos son a grandes magnitudes y afecta principalmente el entorno familiar en el que tiene lugar la desintegración del hogar, bajo nivel educativo de los hijos e hijas, quienes pueden repetir el patrón de comportamiento sobre violencia intrafamiliar y descuido de los niños.

El femicidio en el barrio Cristo Rey ha provocado en las mujeres "temor a que les suceda lo mismo", situación que genera preocupación y refuerza las estrategias en pro de disminuir el femicidio.

Criselda Calero, Sayda González y Anameyling Guevara 
Esto se refleja con el $46 \%$ de las repuestas de las féminas; en tanto, el $29 \%$ de los hombres demostró una mayor preocupación por la comunidad. Algunos manifestaron intranquilidad principalmente en la conducta de los niños que se podría ver influida por estos casos de violencia máxima, así como interés por disminuir el femicidio.

Cabe resaltar que existe un porcentaje del $18 \%$ de los hombres que manifestaron que el femicidio no tiene ninguna afectación para ellos, lo que expresa claramente algunas creencias patriarcales aprendidas de sus padres (Grafica 6).

\section{Conclusiones}

A partir de los resultados encontrados, se llegó a las siguientes conclusiones:

1. Entre las características sociodemográficas más representativas en la población en estudio, se destaca la religión evangélica, el sexo femenino y una escolaridad para la mujer de primaria, y en los varones, de secundaria.

2. La apreciación de los pobladores del barrio Cristo Rey, con respecto a los roles y funciones dentro de la familia vinculados al tema de género, aún está arraigada a la cultura patriarcal, en la que sobresale que las labores del hogar son exclusivamente para mujeres, mientras que la oportunidad de estudiar y jugar es propia de los varones.

3. El maltrato y el femicidio, según la población en estudio, se da por distintos factores, principalmente por "miedo", por "mantener unido el hogar", "por sus hijos" o incluso por "guardar lealtad hacia el hombre".

4. Entre los efectos psicológicos del femicidio, los pobladores del barrio Cristo Rey consideran que estos repercuten mayormente en las mujeres, quienes manifiestan temor a que les suceda lo mismo que las mujeres víctimas de femicidio. Asimismo, indican que en los hijos de las víctimas estos efectos son de desarraigo familiar, social y emocional, quedando vulnerables a un destino incierto que puede afectar muchos ámbitos de su vida.

5. Los hombres consideran que el femicidio puede afectar a la comunidad, implicando un aprendizaje social de conducta desfavorable para los niños, quienes en un futuro podrían repetir el mismo círculo de violencia hacia las mujeres y crear un entorno de inseguridad para todos los pobladores.

\section{Referencias bibliográficas}

1. Bergman, O. (2011). El Machismo mata. Nicaragua .

2. Cuadra, R. (martes de abril de 2014). Nicanoticias.com. Obtenido de Nicanoticias.com: http://www. nicanoticias.com/2014/04/22/matana-maestra-en-la-entrada-del-colegioen-tipitapa/

3. Díez, S. (14 de enero de 2014). mujerAF. Obtenido de mujerAF: http://www. mujeraf.com/2014/01/violenciaeconomica-arma-de-dominacion-ycontrol/

4. Fernanda, M. (20 de abril de 2015). Psyciencia.com. Obtenido de Psyciencia.com: http://www. psyciencia.com/2015/04/20/por-quesimplemente-no-se-va-la-realidad-deescapar-de-la-violencia-domestica/

5. Barcaglione,G. (2005). Femicidios e impunidad. Centro de encuentros.

6. Larrosa, M. P. (11 de diciembre de 2010). Revistas.ucm. Obtenido de Revistas.ucm: http://revistas. ucm.es/index.php/FORO/article/ viewFile/37248/36050

7. Lagarde, M. (2000). Claves feministas para la negociación del amor. México .

8. Zanetti, I. (13 de mayo de 2013). Psyciencia. Obtenido de Psyciencia: http://www.psyciencia. com/2013/05/13/genero-relacionesde-poder-y-subjetividad/ 